\title{
A MONITOR FOR GAMMA RADIATION AT ZERO DEGREES FROM THE SLC COLLISION POINT *
}

\author{
G. BONVICINI, R. FREY, E. GERO and W. KOSKA \\ University of Michigan, Ann Arbor, MI 48109, USA
}

\section{FIELD}

Stanford Linear Accelerator Center, Stanford, CA 94309, USA

\author{
A. MINTEN \\ CERN, CH-1211, Geneva 23, Switzerland
}

Received 21 November 1988

Bremsstrablung gamma rays are emitted in a narrow forward cone while the electron and positron beams are being measured with carbon fibers at the SLC (the linear collider at the Stanford Linear Accelerator Center). Beam-beam deflections which occur at the collision point also emit softer gamma rays, called beamstrahlung. A device to detect these gammas is described. It has a converter to produce electron-positron pairs and a gas Cherenkov volume to detect them in the presence of a large, low energy, radiation background. The system is used for beam diagnostics at the SLC.

\section{Introduction}

The concept of the linear collider is centered on the collision of high intensity beam bunches of microscopic dimensions. The development of diagnostic procedures for the beams and their collisions will be important for the success of this new class of machines. In the case of the SLC, beam transverse dimensions are a few microns, with intensities up to $5 \times 10^{10}$ in bunches a few millimeters long. Measurements on single beams using fine carbon fibers have been described $[1,2]$ and these are now most commonly performed by using the counters described here to measure the bremsstrahlung from the fibers. Also, when the beams are near collision, their fields are intense enough to deflect each other [3], and the resulting radiation may be detected in this device and used to monitor the collisions.

\section{The environment}

Inside the Mark II detector, at the interaction point (IP) of the SLC, is a retractable target of carbon fibers [2]. There are three fibers, $150 \mu \mathrm{m}$ apart, of nominal

\footnotetext{
* Work supported by the Department of Energy, contracts DE-AC03-76SF00515 and DE-AC02-76ER01112.
}

diameters 30,7 and $4.5 \mu \mathrm{m}$. The electrons and positrons collide head-on, and the single beam pipe is in a straight line extending through the last focusing stage of each beam line. The first significant dipole, B1, is encountered by the exiting beam on its way to the dump at about $38 \mathrm{~m}$ from the IP, and by $42 \mathrm{~m}$ the beam has been deflected $6.9 \mathrm{~cm}$. At this point, between beam line elements, there is room for some apparatus outside the vacuum pipe.

The geometry of the beam line components makes it possible to monitor neutral radiation within a cone of half angle $1 \mathrm{mrad}$ about the axis of the beams at the interaction point. The vacuum pipe is sized so that it comfortably contains the $1 \mathrm{mrad}$ cone. At about $20 \mathrm{~cm}$ in front of the counter to be described, however, there is a flange, and the beam, deflected by $B 1$ by about $1^{\circ}$, continues in a pipe of $1.9 \mathrm{~cm}$ diameter (fig. 4). The edge of the counter is parallel to this narrow pipe, at a distance of $1.5 \mathrm{~mm}$. The neutral radiation in the $1 \mathrm{mrad}$ cone escapes from the vacuum tank through a $76 \mu \mathrm{m}$ thick stainless steel window and enters the face of the counter. There is room for only a narrow septum in the flange between the thin window and the $1.9 \mathrm{~cm}$ beam pipe.

The dipole B1 has a field of $1.2 \mathrm{~T}$. As the spent charged beam passes through it, a flux of synchrotron radiation is added to any radiation from the interaction point. The "critical energy" [4] of the spectrum is 2.3 
$\mathrm{MeV}$. For $10^{10}$ beam particles, $25 \mathrm{~mJ}$ illuminates a horizontal stripe across the face of the detector, corresponding to about $200 \mathrm{rad}$. In addition, there is a variable radiation background of gammas and electrons at a few MeV from the nearby showering of off-trajectory beam particles. Scraping upstream can also give rise to a flux of hard photons inside the beam pipe, coincident with the signal from the IP.

\section{Radiation from the IP}

Photons from collisions of a charged beam with a carbon fiber have the $1 / k$ bremsstrahlung spectrum. Their emission cone is effectively identical with the divergence of the charged beam, which is tuned within the range of $100-300 \mathrm{mrad}$ vertically and horizontally. The lowest flux of interest during SLC tuning is about $3 \times 10^{4} \mathrm{\gamma} /$ pulse above threshold in the detector.

The radiation from beam-beam deflection (so-called beamstrahlung) is more difficult to treat. In the neighborhood of each bunch is a high electromagnetic field whose effect on particles in the opposing bunch is to deflect them towards its axis. The resultant radiation under SLC conditions is effectively synchrotron radiation with a range of critical energies, $E_{\mathrm{c}}$. The mean critical energy can be from some $\mathrm{MeV}$ to some hundreds of $\mathrm{MeV}$, depending on beam conditions.

In the case of round beams with Gaussian distributions, equal dimensions, and colliding head-on, the radiated energy is [5]

$W_{1}=0.22 r^{3} \gamma^{2} m c^{2} N_{1} N_{2}^{2} / \sigma_{r}^{2} \sigma_{z}$,

where $N_{1}, N_{2}$ are the radiating and target beam populations, and the $\sigma$ represent the Gaussian dimensions of the beams. The constants are: $r$ and $m$, the classical radius and mass of the electron, $\gamma$ the Lorentz factor, and $c$ the velocity of light. If $L$ is luminosity per collision,

$W_{1} \propto L N_{2} / \sigma_{z}$.

However, when the collisions are not head to head, luminosity falls off faster than does the beamstrahlung intensity. For cases where all the beam dimensions are different, analytic estimates are also difficult. For this reason, simulation programs have been employed [6] and they have the advantage of permitting calculations to be applied specifically to the spectral range relevant to a given counter configuration. Examples of simulation results are shown in figs. 1 and 2 . In the first, the signal from a counter, similar to that described below, is plotted against the impact distance between two beams of the same size and intensity, as one of them is steered, pulse by pulse, on either side of the collision point with

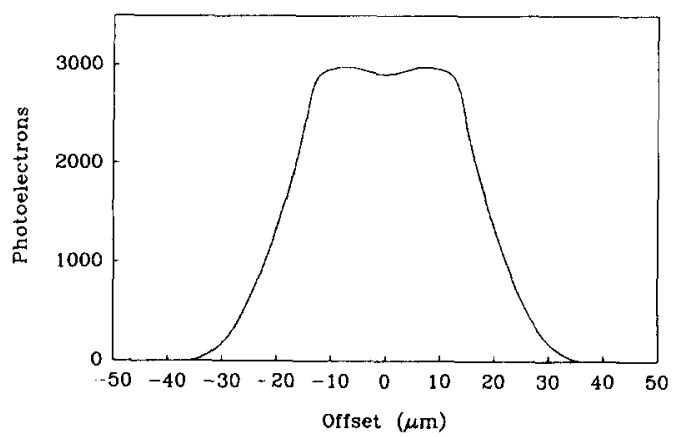

Fig. 1. Simulation results: Expected beamstrahlung yield, expressed in photoelectrons from the photomultiplier tubes, as the position of one beam is offset relative to the other. The Gaussian beams have a radius of $6 \mu \mathrm{m}$, length $0.5 \mathrm{~mm}$, and intensity $10^{10}$ per pulse.

the other. Fig. 2 shows the counter's photoelectron yield, for head-on collisions, versus beam size. The luminosity scale is also shown, assuming the nominal SLC operating frequency of $120 \mathrm{~Hz}$. In fact, conditions of interest at SLC correspond to fluxes of $10^{5}$ up to more than $10^{10}$ gammas with energy above $20 \mathrm{MeV}$.

Each of these gamma rays also has a very small emission angle. It follows that the angular distribution of the radiation is the same as that of the charged beam during the collision. Deflections of the two charged beams conserve transverse momentum and so are coplanar, but of opposite sense in the transverse plane. The same therefore applies to the radiation. The mean angle of the radiation, relative to the head-on collision case, is just half as much as for the charged beam deflection. In addition, even in the case of head-on collisions, the beams tend to disrupt each other, increasing the size of the divergence cones. This effect may be measurable using the radiation cones at high luminosities.

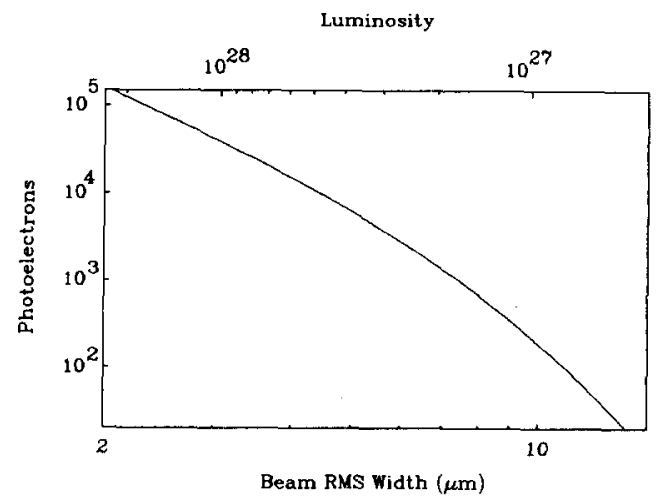

Fig. 2. Simulation results: Expected beamstrahlung yield, in photoelectrons, against the beams' radial size for bunch populations of $10^{10}$. The luminosity scale is also given, assuming the SLC nominal repetition rate of $120 \mathrm{~Hz}$. 


\section{Counter description}

Because of the enormous flux of low energy radiation, the detector was designed to be sensitive only above an energy threshold in the neighborhood of 20 $\mathrm{MeV}$. The relationship of the synchrotron radiation spectrum and that of the beam-beam deflection radiation is illustrated in fig. 3 . The bremsstrahlung spectrum, of course, extends up to the charged beam energy.

The selection of a Cherenkov device afforded a good rejection of background even when tuned for low thresholds, yet good acceptance for the broad bremsstrahlung spectrum. Additionally, a compact design was possible - room is very constrained in the tunnel on the north side of the IP.

The design makes use of a converter plate to produce electron-positron pairs from a fraction of the gamma rays. A gas volume with selectable pressure follows, and Cherenkov light from the electrons and positrons above threshold is collected and imaged onto an array of photomultiplier tubes.

The converter wall is, in fact, part of the stainless steel pressure vessel, and converts $3 \%$ of gammas at 50 $\mathrm{MeV}$. Because the energies of the emerging electrons and positrons from beamstrahlung are so low, the converter was kept thin to reduce losses from severe multiple scattering. For bremsstrahlung studies, a 3 r.l. plate of lead is remotely moved into place in front of the counter, amplifying this high energy signal without consequences from multiple scattering.

The synchrotron radiation photons have a probability of Compton scattering in the Cherenkov gas, and the scattered electrons can cause scintillation. In order to minimize this, the counter is filled with ethylene [7]. If nitrogen were to be used, the resulting background would be equivalent to the beamstrahlung at a luminosity of $10^{29} \mathrm{~cm}^{-2} \mathrm{~s}^{-1}$. Since electron thresholds in the

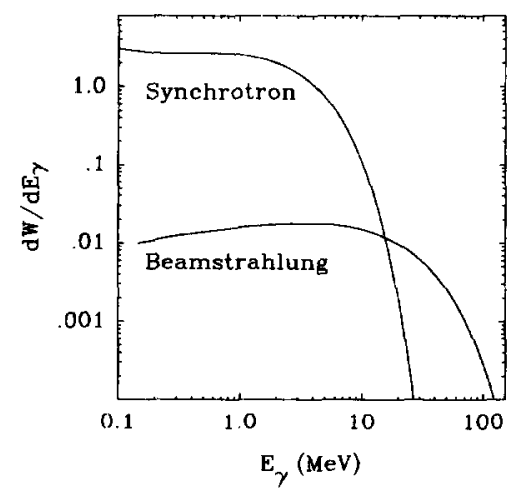

Fig. 3. Comparison of energy spectra of synchrotron radiation and beamstrahlung. Beam intensities are set at $10^{10}$ per bunch, with Gaussian distributions of rms radius $4 \mu \mathrm{m}$ and rms length $1 \mathrm{~mm}$.

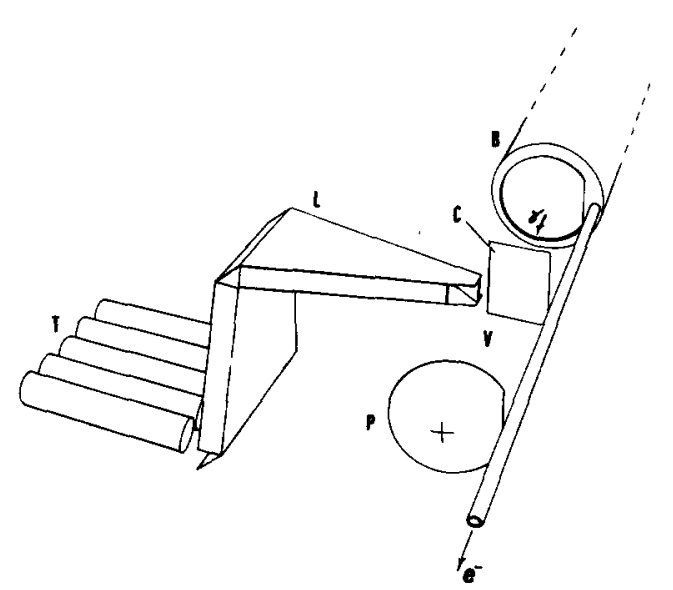

Fig. 4. Layout of the optical components of a counter in relation to the beam line. The gas containment vessel is not shown. B: large diameter beam pipe, flange, thin stainless steel window; $\mathrm{C}$ : converter plate; V: Cherenkov emission volume; P: primary mirror; L: light channel, with periscopic reflections; T: photomultiplier tubes and fused silica vacuum windows.

range of $20 \mathrm{MeV}$ are needed, it follows that the counter must be operated at pressures below atmospheric.

The effects of multiple scattering in the converter plate are largely eliminated by using optics which image the converter onto the light detectors. Fig. 4 illustrates the optical layout and the geometry of the counter. The design was intended to suppress the scattering of radiation into the light detectors, and to facilitate effective shielding.

A $21 \mathrm{~cm}$ Cherenkov emission path ends at the field mirror. This element, which must withstand the full intensity of the synchrotron radiation, was electroformed of nickel, and uses a reflecting coat of rhodium. It is $2.5 \mathrm{~mm}$ thick, has an optical radius of $33 \mathrm{~cm}$ and an aperture of $13.7 \mathrm{~cm}$. It directs the light back through a waist at $17 \mathrm{~cm}$, where an iris is formed from the next optical components.

A plane mirror reflects the light away from the beam line. As a step towards reducing the scattered radiation reaching the light detectors, the area needed to transmit the light to the image plane was reduced. This is done by channeling it between parallel reflector plates $3.2 \mathrm{~cm}$ apart. These were fabricated from prepolished stainless steel, vacuum coated with aluminum. In addition, two $90^{\circ}$ reflections are used as a periscope to allow for more effective shielding.

As laid out in fig. 4, this arrangement condenses the vertical projection of the image, and so the vertical angular distribution of the gamma radiation cannot be observed. On the other hand, the horizontal projection is preserved. It is observed by imaging it on to a set of five photomultiplier tubes, side by side, outside the gas volume. 
Photomultiplier tubes were selected as the light detectors because they are not particularly sensitive to the very low energy ionizing radiation in the area. Still, massive shielding was necessary to suppress the incidence of photons which could scatter in or near the faces, producing electrons above $160 \mathrm{keV}$, the Cherenkov threshold in the glass in front of the photocathodes.

In practice, the ability to measure the horizontal distribution was implemented for the counter which observes radiation from the electron beam. The other counter was rotated to measure the vertical divergence of the positron beam. In the plane perpendicular to the beam axis, the charged beam deflections are in opposite directions, and so this arrangement permits both horizontal and vertical components of deflection to be monitored. It is thus possible to track targeting offsets between charged beams which are nominally in collision.

The optical system is enclosed within a stainless steel pressure vessel. This was designed to minimize access for scattered radiation into the photomultiplier tubes, while supporting the optics rigidly and permitting massive lead shielding to be built around them. Flange seals were made using indium wire gaskets. The light leaves the vessel through a standard vacuum window of fused silica in a stainless steel jacket, in front of each PMT.

The PMTs, in low permeability magnetic shielding, are protected by a steel box from the mass of the lead shielding. An LED has been placed to allow checks of each tube's performance.

The counters and their shielding are supported from the floor, and positioned within $2 \mathrm{~mm}$ of the narrow beam pipe. Because of the fear of alignment complications, this has been done without touching the beam line girder. Roughly 1.5 tons of lead were stacked around the PMTs and the light channels, with particular emphasis on absorbing radiation from identifiable

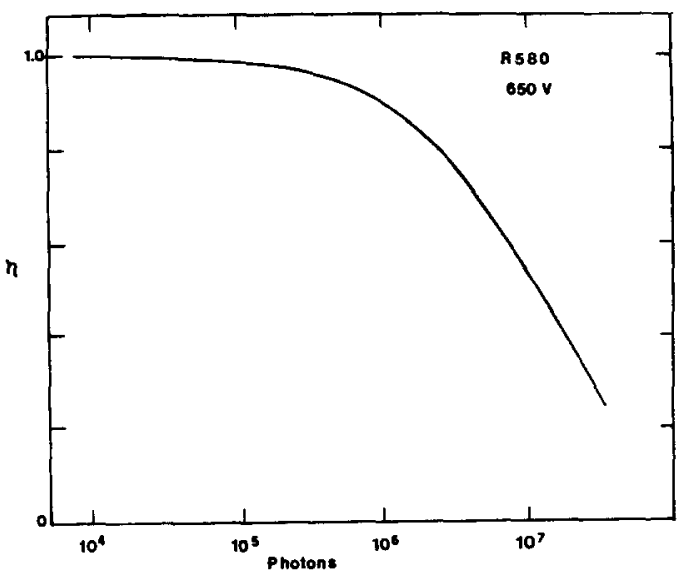

Fig. 5. Saturation curve: photomultiplier tube efficiency, $\eta$, against incident light pulse amplitude. scattering sources, for example the flange between the thin vacuum window and the narrow beam pipe.

The gas system is accessible at surface level. It flows commercial purity ethylene through a vacuum regulator to the copper lines going to the counter vessel. The return line flow is controlled by a needle valve, before the gas enters a vacuum reservoir. This reservoir is serviced by an oil sealed rotary vane pump. There are, of course, pressure sensors and shutoff valves appropriate for hazardous gas operation. The volume in the tunnel is very small, and at negative pressure, and is not considered a special hazard.

The photomultiplier tubes, Hamamatsu R580 [8], were chosen, from among several types tested, for their ability to deliver high instantaneous currents. This maximizes the dynamic range of the signals detectable above the cable noise level (about $100 \mu V$ at present). Tube gains in the general range up to $10^{4}$ are adequate.

The anode sensitivity and saturation characteristics of each tube were measured, using a light integrating box with controllable apertures and calibrated filters, and intense nanosecond pulses from a nitrogen laser. The short pulses simulated the sharp Cherenkov light timing. In fig. 5 is illustrated the space charge saturation curve measured for a typical tube. Note that when the SLC luminosity improves beyond $10^{29} \mathrm{~cm}^{-2} \mathrm{~s}^{-1}$ the tubes will operate partially saturated. It will be desirable to replace them at that stage, and we have found that mesh dynode tubes would eliminate the saturation problem. The voltage divider base design is of the nonlinear type, to optimize instantaneous current delivery. Components were chosen for radiation resistance.

The tubes for each counter were selected for similar sensitivity. Final balancing was done by adjusting high voltage settings, using a simple, stable and reliable resistive divider network.

\section{Signal processing}

Signals from the anode and last dynode of the PMT bases are taken, on RG214 cables, from the SLC tunnel to tha surface. The dynode signals are inverted and digitized by an LRS [9] $2249 \mathrm{~W}$ ADC, from the SLC standard inventory. The anode signals are amplified by a factor of 20, using a Phillips [10] 7177, before digitization. This amplification effectively broadens the dynamic range of the system beyond the 11 bits of a single ADC channel. The 50 ns wide gate is derived from the SLC computer controlled timing system.

For every SLC pulse, the digitized data are transmitted over a CAMAC link to a Multibus-based microcomputer using an Intel 8086 microprocessor. This is also a standard SLC unit normally used for local control and feedback. In this case, the data are acquired 
in the form of four sets of five ADC results, i.e. the sets of five PMTs for both the electron and positron beam provide results at the two levels of amplification.

Pulse by pulse, the microcomputer calculates the sum of the amplitudes, and also the center and width of the beam spot, using the first and second moments of the pulse height distributions of each set of five signals. Also on every pulse, the data and calculations are made available to a PC AT class microcomputer, by way of a dual port memory unit resident on the Multibus. The latter computer is used as a monitor and diagnostic device.

Results are transmitted from the Multibus computer when the SLC central computer requests them. In addition, the Multibus computer can subtract pedestals or backgrounds from the data, decide by itself whether to send results from amplified or unamplified channels, or comply with a demand for one or the other, as instructed by the central computer. In this way the central computer has pulse by pulse access to the counter's signals as required, at rates up to $120 \mathrm{~Hz}$. Real-time correlations between pairs of synchronous variables such as beam steering versus counter pulse amplitude can be selected, and are plotted, on request, as a standard feature of the SLC operating program SCP [11].

In addition, the data are made available, pulse by pulse, in a cyclic buffer which can be accessed asynchronously by the central computer.

\section{Operational experience}

Beam tuning exercises at the interaction point frequently make use of the thin fiber target to establish the effect on the spot size of altering beamline parameters. This is done by steering the beam in steps of one or a few microns across the fiber. The resulting bremsstrahlung intensity is the overlap function of the beam and fiber shapes.

The 3 r.l. converter plate has normally been used to amplify the signal, and the detector has been operated at $0.33 \mathrm{~atm}$. The threshold momentum for electrons was consequently $25 \mathrm{MeV} / c$.

By reading back the summed pulse heights after every pulse, the SLC control computer is able to correlate the bremsstrahlung intensity with the beam steering control. An example of beam profile plots from a single scan of the beam across three fibers is found in fig. 6 . Signals from a $4.5 \mu \mathrm{m}$ fiber can be seen clearly in the figure. Despite being relatively small, they will become important when SLC spot sizes are reduced below 2.5 $\mu \mathrm{m}$. Beams with Gaussian widths as narrow as $2.8 \mu \mathrm{m}$ have been measured even with a $7 \mu \mathrm{m}$ fiber, but the beam width, convoluted with the mass distribution of the fiber, was observed as $3.3 \mu \mathrm{m}$.

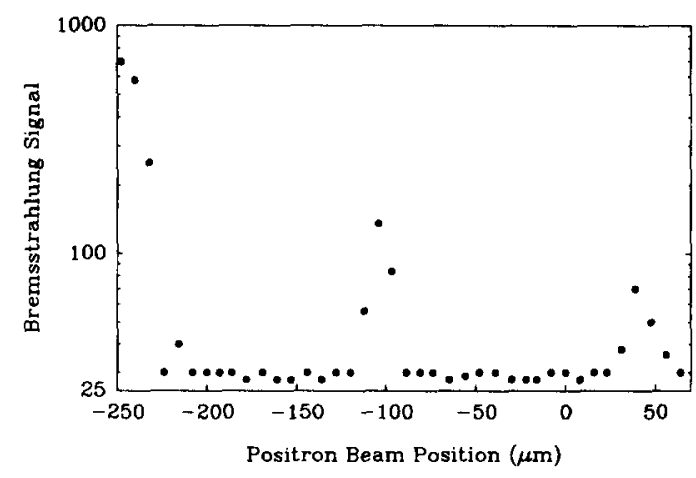

Fig. 6. Example of the response of a counter to the scanning of a positron beam across carbon fibers at the collision point. Signals from the edge of the $30 \mu \mathrm{m}$, and from the 7 and $4.5 \mu \mathrm{m}$ fibers can be seen above ADC pedestal level. The fibers are $150 \mu \mathrm{m}$ apart.

The backgrounds under the illustrated profiles are reasonably flat. This has not always been the case. Especially in early phases of the development of the SLC, the scraping of the beam tails, far upstream of the counter, has occasionally given rise to a substantial bremsstrahlung background in the beam pipe. It has, however, been possible to measure beam dimensions with backgrounds even larger than the signal from the fiber, provided that the fluctuations were not too rapid.

When the beam is focussed some centimeters upstream or downstream of a fiber, and it is scanned across the fiber, only a fraction of the phase space is intercepted by the fiber on any given pulse. Beam trajectories from only a small range in angles can hit the fiber, and so the angular range of the bremsstrahlung is also restricted. As the charged beam is scanned across the fiber, the bremsstrahlung sweeps like a lighthouse beam. Fig. 7 illustrates the response of one of the counters to this phenomenon. The signals from each of the tubes, and their sum, are plotted pulse by pulse. It can be seen that the gamma ray beam sweeps across the counter in approximately 8 pulses. The mean emission direction is also plotted every pulse. The rms angular width of the total flux can be obtained from the righthand part of the figure, and is $322 \mu \mathrm{rad}$. The average angular width from individual pulses is 280 , with fluctuations of up to $20 \mu \mathrm{rad}$.

By examining the level of fluctuations about the rate of sweep of the mean angle, it has been possible to show that the counter's precision for measuring beam deflections is better than $20 \mu \mathrm{rad}$, at least when the signal exceeds the background and pedestal fluctuations by a factor of about 5 .

The number and spacing of the PMTs have been chosen, after Monte Carlo studies, to limit to the $5 \mu \mathrm{rad}$ level the effects of geometry on the measurement of the mean emission direction of the radiation. For signals of 


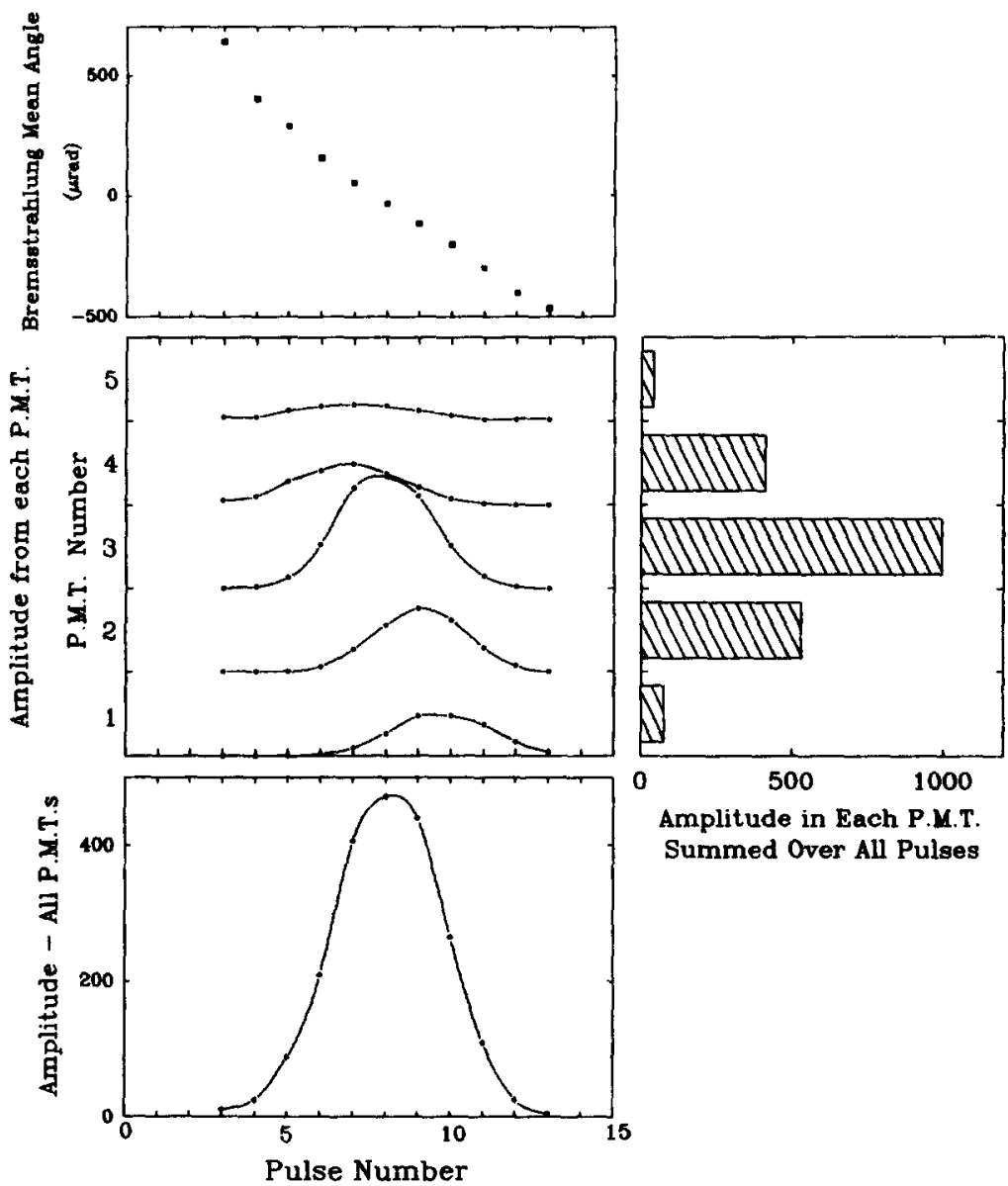

Fig. 7. Illustration of the response of the individual PMTs as an out-of-focus beam is swept, pulse by pulse, across a fiber. The signals from the two outer PMTs are shown scaled by a factor of 5 , in the center part of the figure only, to make them clearer. Amplitudes are in $\mathrm{ADC}$ counts.

interest, the number of gamma rays, of pairs, and of photoelectrons all exceed 1000 , and statistical uncertainty is also below the $5 \mu \mathrm{rad}$ level. However, pulseto-pulse fluctuations in the angle of the charged beam are larger than this, and it has not yet been possible to implement corrections using beam position monitor readings. In addition, the effects of pedestal and background fluctuations on small signals require further study. Resolution also deteriorates, because of leastcount uncertainty, when the digitized signal size is below 50 counts.

The narrowest gamma ray "beams" observed so far have had indicated widths of about $180 \mu \mathrm{rad}$. The width resolution expected for a bremsstrahlung signal is approximately $150 \mu \mathrm{rad}$, dominated by the effect of the PMT spacing, with contributions from the thickness and spacing of the lead converter from the face of the counter, and from the dead space between the PMTs.

The range of observations with carbon fiber targets indicates that the counters are performing as designed.
Given sufficiently low levels of background, they should be able to monitor the collisions of the SLC beams. Indeed, as the SLC development work has proceeded, radiation signals from the collisions have increased by

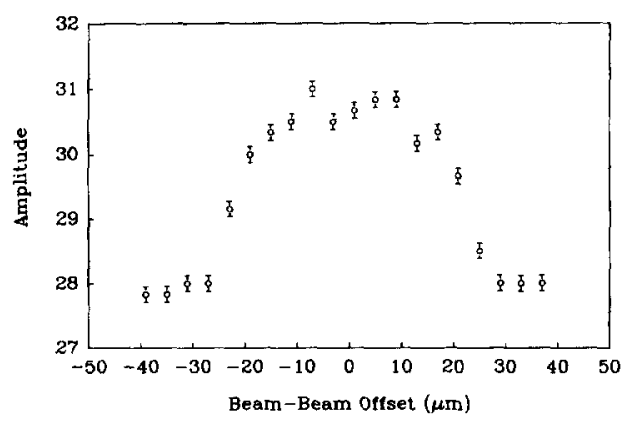

Fig. 8. Example of beam-beam deflection radiation. Counter response is plotted as one beam is steered across the other at the collision point. Signal amplitude is in ADC units, and six pulses are averaged for each $4 \mu \mathrm{m}$ step in the abscissa. 
much more than an order of magnitude. As shown in fig. 8, clear signals are observed above pedestal and background when the positron beam is scanned across the position of the electron beam at the IP. As discussed above, the particles in one beam are deflected by the other when the bunches are close by, and during the deflection the particles radiate. Such scans consequently give rise to a characteristic peak of beamstrahlung whose exact shape depends on the relative dimensions of, and offsets between, the two primary beams. The intensity has not yet been strong enough, nor the deflection large enough, to measure the beam deflection using the gamma ray counter. But the intensity measurement has been brought into use to help tune the beams in collision.

\section{Summary}

A pair of gamma ray detectors for zero degree emission from the SLC collision point has been built and brought into operation in a hostile radiation environment. The counters serve together with the carbon fiber targets as beam profile monitors for the collision point. They have now also come into use as monitors of the collision of the electron and positron beams.

\section{Acknowledgements}

We wish cordially to acknowledge the interest of Prof. J. Ballam and Dr. W. Kozanecki, and thank them for their efforts on behalf of this project. That the SLC can deliver micron-size beam spots with backgrounds tolerable to these counters is because of the work of many dedicated people, too numerous to mention here. In particular, however, we thank Dr. Nan Phinney for her help with the data collection and the SLC software. Valuable contributions were made by K. Bouldin, particularly to the work with the photomultipliers. We also wish to thank Dr. R. Erickson for facilitating the integration of the equipment into the SLC system, the SLAC vacuum group for fabricating the beam pipe with its thin window, and A. Gromme and L. Sanchez for dedicated work with the SLC software.

\section{References}

[1] R. Fulton et al., Nucl. Instr. and Meth. A274 (1989) 37.

[2] G. Bowden et al., SLAC-PUB-4744 (Nov. 1988), submitted to Nucl. Instr. and Meth.

[3] P. Bambade and R. Erickson, SLAC-PUB-3739 (May 1986).

[4] M. Sands, SLAC-121 (November 1970) p. 116.

[5] M. Bassetti et al., IEEE Trans. Nucl. Sci. NS-30 (1983) 2182.

[6] Simulation code for this project was developed from a beam disruption simulation program described in: $\mathbf{R}$. Hollebeek, Nucl. Instr. and Meth. 184 (1981) 133; see also G. Bonvicini, C. Field, A. Minten, Mark II SLC Note 220, SLAC (April 5, 1985).

[7] J. Heintze et al., Nucl. Instr. and Meth. 138 (1976) 641.

[8] Hamamatsu Photonics KK, Hamamatsu City, Japan.

[9] LeCroy Research Systems Inc., Spring Valley, NY, USA.

[10] Phillips Scientific, Suffern, NY, USA.

[11] N. Phinney, IEEE Particle Accelerator Conf. Proc. (1985) p. 2117. 